\title{
87. Infectivity of the Cloned DNAs from Multiple Genome Components of Bean Golden Mosaic Virus
}

\author{
By Tsutou Morinaga,*) Masato IKegami,**) \\ and Kin-ichiro MIURA*) \\ (Communicated by Motoo Kimura, M. J. A., Dec. 12, 1983)
}

Bean golden mosaic virus (BGMV), which is a twinned spheres shape and contains a small, circular, single-stranded (ss) DNA as a genome, is classified into geminiviruses. Electronmicroscopic observation showed that the size of circular ssDNA from a BGMV preparation is about $0.9 \mu \mathrm{m}$ (2500 nucleotides long). ${ }^{1 \prime}$ However, digestion of BGMV ssDNA with ssDNA specific endonucleases, Hae III or Hha I, showed that the total size of BGMV-DNA is about 5000 nucleotides long. ${ }^{2)}$ These observations and infectivity-dilution kinetics ${ }^{2)}$ suggest that a genome of BGMV consists of two kinds of circular ssDNA of almost similar length around 2500 nucleotides. Here, it is reported that the presence of such two DNA molecules was proved by cloning these DNAs, and two DNA molecules were required for revealing infectivity. Besides two DNA components, a minor component being similar in size was also found.

BGMV was isolated from the infected leaves of Phaseolus vulgaris L., “Top Crop". DNA was extracted and purified by treatments with proteinase $\mathrm{K}$ and phenol containing SDS. The viral ssDNA was converted to double-stranded (ds) DNA by AMV reverse transcriptase with primers, which were oligonucleotide mixture obtained by complete digestion of calf thymus DNA with DNase I. In this step, $\left[\alpha{ }^{32} \mathrm{P}\right] \mathrm{dCTP}$ was used to label the DNA. The dsDNA was digested with several kinds of restriction endonucleases, and analyzed by electrophoresis and radioautography. Most of the fragments were classified into two groups by their density. The total size of the thicker fragments is similar to that of the thinner fragments, corresponding to the size of circular ssDNA observed by electronmicroscopy. Besides these groups, much more thinner fragments were detected in amounts of ca. $4 \%$ of total DNA in gel electrophoresis. The total size of these minor DNA fragments is also about 2600

*) Department of Applied Chemistry, Faculty of Engineering, University of Tokyo, Hongo, Tokyo 113; National Institute of Genetics, Mishima 411.

**) Nodai Research Institute, Tokyo University of Agriculture, Sakuragaoka, Setagaya, Tokyo 156. 
nucleotides long as the major DNAs. These results lead us to the conclusion that there are two major DNA molecules (DNAs I and II), and at least one minor DNA molecule (DNA III) in BGMV preparations. Based on these analysis by endonuclease digestion, physical maps of two major DNAs are deduced as shown in Fig. 1.
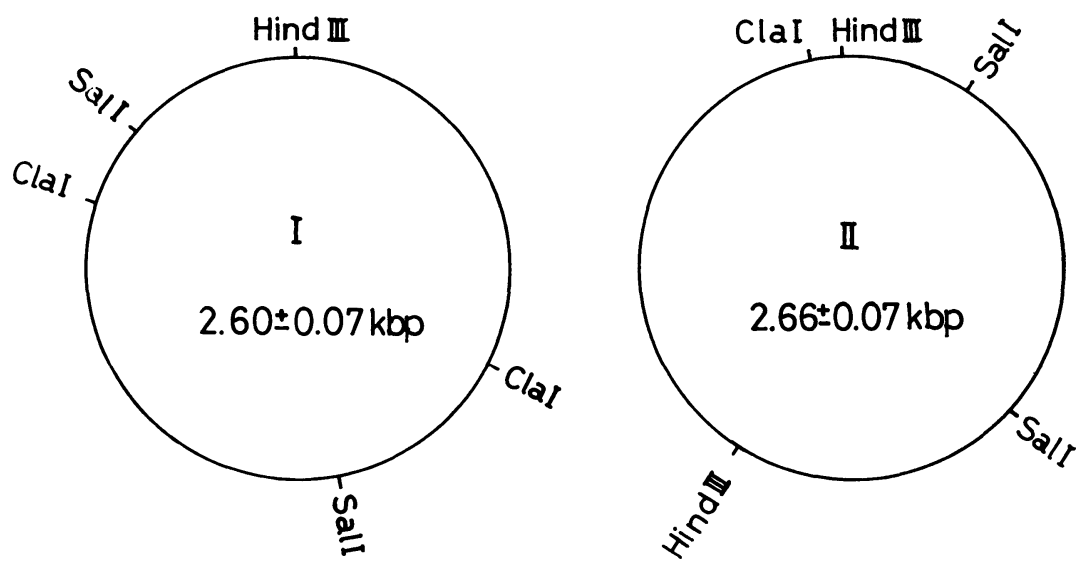

Fig. 1. Physical maps of BGMV DNA.

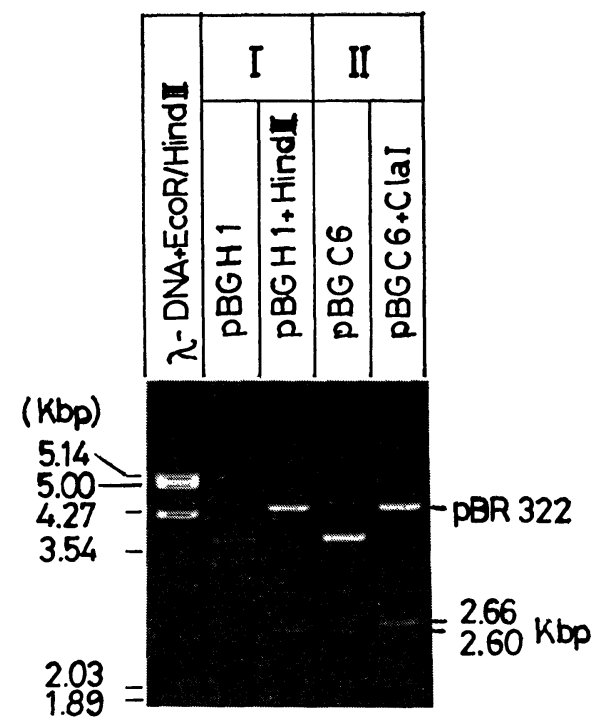

Fig. 2. Gel electrophoresis of cloned BGMV DNA.

The dsDNAs of BGMV were cut at one site by restriction endonucleases, Hind III for DNA I, Cla I for DNA II, and cloned with the use of a vector plasmid pBR 322 in E. coli HB101 (Fig. 2). Endonuclease analysis of the cloned DNAs I and II confirmed the abovementioned physical maps. 
As either DNAs I or II was cloned, they were prepared in large scale each other, and their infectivity for a host plant was tested. The cloned DNA I in pBR 322 (pBGH1) was digested with Hind III, and DNA II in pBR 322 (pBGC6) with Cla I to yield a linear dsDNA fragment corresponding to the full size of an ssDNA component from a virus preparation. DNA preparations were rubbed on the primary leaves of Top Crop with Celite powder. Infectivity of DNA preparations is summarized in Table $I$. When dsDNA I (the Hind III

Table I. Infectivity of cloned BGMV DNA

\begin{tabular}{l|ccc}
\hline \multicolumn{1}{c|}{ DNA source } & $\begin{array}{c}\text { Amount } \\
(\mu \mathrm{g})\end{array}$ & infected $/$ inoculated* \\
\hline & 20 & Experiment 1 & Experiment 2 \\
\hline $\begin{array}{l}\text { BGMV ssDNA } \\
\text { (Viral DNA) }\end{array}$ & 10 & $3 / 3$ & - \\
$\begin{array}{l}\text { pBGH1+Hind III } \\
\text { (DNA I) }\end{array}$ & 20 & $3 / 3$ & - \\
& 10 & $0 / 3$ & - \\
pBGC6+Cla I & 10 & $0 / 3$ & $0 / 11$ \\
(DNA II) & 10 & $0 / 2$ & $0 / 11$ \\
pBGH1+Hind III & + & & $7 / 11$ \\
and \\
pBGC6+Cla I \\
(DNA I+DNA II)
\end{tabular}

* Infectivity was tested by rubbing the shown amount of DNA on expanding primary leaves of Top Crop. About 2 weeks after inoculation symptoms of golden yellow mosaic appeared.

digest of $\mathrm{pBGH1}$ ) or dsDNA II (the Cla I digest of pBGC6) was inoculated separately, no symptom was observed on the leaves during 2 weeks. On the other hand, when the mixture of dsDNAs I and II was used, distinct infection was caused as the case of a viral DNA preparation. Linear dsDNAs, if a mixture of I and II, showed infectivity as circular ssDNAs from a virus preparation. The informations necessary for virus multiplication seem to be divided in DNAs I and II, but not in a minor component, DNA III. Details of the structural study on the genome of BGMV will be reported elsewhere.

The authors acknowledge Dr. Kunitada Shimotohno for instruction for DNA cloning, and Dr. Taro Fujii, Mr. Takashi Yoshida, and Mr. Motoharu Takeuchi for cultivation of bean plants. 


\section{References}

1) Reisman, D., Riccardi, R. P., and Goodman, R. M. (1979): Virology, 97, 388-395.

2) Haber, S., Ikegami, M., Bajet, N. B., and Goodman, R. M. (1981) : Nature, 289, 324-326. 